\title{
Early retinal neurodegeneration in preclinical diabetic retinopathy: a multifactorial investigation
}

\author{
Ibrahim Toprak $\mathbb{D}^{1} \cdot$ Semin Melahat Fenkci ${ }^{2} \cdot$ Guzin Fidan Yaylali ${ }^{2} \cdot$ Cigdem Martin $^{1} \cdot$ Volkan Yaylali $^{1}$
}

Received: 21 April 2019 / Revised: 3 July 2019 / Accepted: 9 September 2019 / Published online: 25 October 2019

(c) The Author(s), under exclusive licence to The Royal College of Ophthalmologists 2019

\begin{abstract}
Background/Objectives To investigate effects of microalbuminuria (MA), diabetes duration, glycosylated haemoglobin (HbAlc) level, hypertension (HT) and/or hyperlipidaemia (HL) coexistence on retinal layers in diabetic patients without diabetic retinopathy (DR) using spectral-domain optical coherence tomography (SD-OCT).

Subjects/Methods This cross-sectional study involved 95 (45 had MA and 50 had no MA) patients with type 2 diabetes mellitus (DM) without DR and 91 age- and gender-matched non-diabetic controls. Macular and peripapillary SD-OCT measurements (Heidelberg Engineering GmbH, Heidelberg, Germany), DM duration, HbA1c levels and presence of HT and/or HL were used for statistical analyses.

Results The MA (+), MA ( - and control groups had similar age and gender distribution $(p>0.05)$. The differences in SDOCT measurements among the MA $(+)$, MA $(-)$ and control groups were insignificant $(p>0.05)$. However, diabetic patients $(n=95)$ had significantly thinner inferior-temporal peripapillary retinal nerve fibre layer (RNFL) $(p=0.042)$ than in the controls $(n=91)$. Superior peripapillary RNFL was significantly thinner in patients with an HbA1c level $>7 \%(p=$ 0.049). However, $3 \mathrm{~mm}$-nasal, temporal and superior perifoveal thicknesses were significantly lower in patients with DM duration over 10 years $(p<0.05)$. HT and/or HL coexistence did not lead a significant difference in SD-OCT parameters among the groups.

Conclusions In diabetic patients without DR, peripapillary inferior-temporal RNFL thinning might be an early sign of neuroretinal degeneration and it seems to be independent from vascular endothelial damage (MA). Poor metabolic control appears to lead superior peripapillary RNFL thinning, while perifoveal thicknesses tend to decrease with longer DM duration.
\end{abstract}

\section{Introduction}

Diabetes mellitus (DM) nearly affects every tissue in human body. It is well known that chronic hyperglycaemia causes activation of pathological molecular pathways that result in excessive inflammatory mediators, oxidative stress and release of various growth factors. All these factors are supposed to play role in pathogenesis of DM complications

Ibrahim Toprak

ibrahimt@doctor.com

1 Department of Ophthalmology, Faculty of Medicine, Pamukkale University, Denizli, Turkey

2 Department of Endocrinology and Metabolism, Faculty of Medicine, Pamukkale University, Denizli, Turkey such as retinopathy, nephropathy, neuropathy and cardiovascular system involvement [1-4].

Microalbuminuria (MA) is concluded to be an earliest sign of diabetic nephropathy and mainly caused by damage to the renal capillary endothelium and podocytes. More importantly, MA is accepted to be biomarker of generalized vascular endothelial dysfunction and increased risk for cardiovascular disorders [5-7].

Diabetic retinopathy (DR) is another important microvascular complication of DM. Clinical diagnosis, staging and treatment of DR are determined according to the vascular and hemodynamic parameters. However, in recent years, retinal neurodegeneration has been suggested to be present in diabetic retinal disease. This theory was supported by the previous electrophysiological and experimental studies showing Muller cell activation, neuronal apoptosis and selective thinning in inner retinal layers in patients with DM [8-10]. 
From a clinical perspective, spectral-domain optical coherence tomography (SD-OCT), as a non-invasive technology, is being used to investigate evidence of retinal neurodegeneration in diabetic patients, and several studies reported thinning in inner retinal layers in patients DM.

The rationale of this current study was to present an extensive multifactorial analysis including MA, hyperlipidaemia (HL) and/or hypertension (HT) coexistence, DM duration and $\mathrm{HbA} 1 \mathrm{c}$ level on neuroretinal alterations in macular and peripapillary regions using SD-OCT in diabetic patients without DR (preclinical DR). At present, there is only one specific study investigating relations between MA and retinal layers in the current literature to best of our knowledge [11].

\section{Methods}

The tenets of the declaration of Helsinki were followed and Local Ethics Committee approved the study methodology. Assuming an effect size $(d)$ of 0.8 at $80 \%$ power and $95 \%$ confidence level, sample size was calculated and found to be at least 41 cases per group $\left(\mathrm{G}^{*}\right.$ Power version 3.1.9.2 computer software, Universität Düsseldorf, Germany). All participants provided written informed consent.

\section{Study group}

This cross-sectional study consisted of 95 patients with type 2 DM (diagnosed at the Department of Endocrinology and Metabolism based on relevant laboratory tests) and age- and gender-matched 91 non-diabetic healthy controls. Forty-five diabetic patients had MA and 50 subjects were MA negative. Persistent excretion of 30-300 mg of albumin in 24-h urine collection was considered as MA positivity.

Subjects with a history of ocular trauma, intraocular surgery, DR (any stage), maculopathy, previous retinal laser treatments, high myopia, glaucoma or optic neuropathy and any kind of media opacity or refractive error affecting OCT quality were excluded from the study.

All subjects underwent a complete ophthalmological examination (including visual acuity measurement, slitlamp biomicroscopy, intraocular pressure measurement using Goldmann applanation tonometry, dilated fundus examination with a non-contact +90 dioptre lens), SD-OCT imaging (Spectralis OCT, Heidelberg Engineering $\mathrm{GmbH}$, Heidelberg, Germany) and fundus angiography (in required cases). DM duration, glycosylated haemoglobin (HbA1c) levels and diagnosis of HT and HL were also noted.

\section{SD-OCT imaging}

A single, experienced technician acquired SD-OCT images of the participants after pupillary dilation. High quality scans $(Q$ score $\geq 18$ ) without artefacts were used for statistical analysis. The macular thickness values were obtained on the basis of the early treatment DR study (ETDRS) rings, which were automatically placed by the SD-OCT software. Macular volume $\left(\mathrm{MV}, \mathrm{mm}^{3}\right)$, central volume $\left(\mathrm{CV}, \mathrm{mm}^{3}\right)$, central foveal thickness (CT, microns), inferior (IT), superior (ST), nasal (NT) and temporal (TT) macular thicknesses (microns) within the $3 \mathrm{~mm}$ ETDRS circle were recorded. In addition, peripapillary inferior retinal nerve fibre layer (IRNFL), superior (SRNFL), nasal (NRNFL), temporal (TRNFL), global (GRNFL), inferior-nasal (INRNFL), inferior-temporal (ITRNFL), superior-nasal (SNRNFL), superior-temporal (STRNFL) thicknesses (microns) were measured.

\section{Main outcome measures}

Right eyes of the all subjects were used for the statistical analysis. Initially, all SD-OCT parameters, age, DM duration and HbA1c level (for only diabetic patients), were compared among the MA (+), MA (-) and control groups using a variance analysis. Moreover, SD-OCT parameters were also compared after the diabetic patients were grouped according to the HbA1c level ( $\leq 7 \%$ vs. $>7 \%$ ), DM duration ( $\leq 10$ years vs. $>10$ years) and existing comorbidities (HT and/or HL).

\section{Statistical analysis}

Statistical Package for Social Sciences (SPSS) version 21 (IBM SPSS Statistics Inc., Chicago, IL, USA) software was used for statistical analysis. The Shapiro-Wilk or Kolmogorov-Smirnov test was used to evaluate normality of the variables. Categorical data were compared between the groups using the chi square test. Quantitative variables (age, DM duration, HbA1c levels and SD-OCT measurements) were presented as mean \pm standard deviation. The one-way ANOVA test with a post hoc Bonferroni correction or independent samples $t$-test were used for comparison of quantitative variables according to the number of groups when parametric test assumptions were met. Otherwise, Kruskal-Wallis or Mann-Whitney U tests were performed. At $95 \%$ confidence interval, a $p$ value lower than 0.05 was accepted as statistically significant.

\section{Results}

The study included 95 patients (95 eyes) with a diagnosis of type 2 DM and 91 non-diabetic healthy control subjects (91 eyes). None of the diabetic patients had DR. Forty-five diabetic patients had MA, whereas remaining 50 subjects were MA negative. There were no significant differences in 
Table 1 Age, gender distribution, DM duration and HbA1 levels in the microalbuminuria $(+)$, microalbuminuria $(-)$ and control groups

\begin{tabular}{|c|c|c|c|c|c|c|c|}
\hline & \multicolumn{4}{|l|}{ DM group } & \multicolumn{3}{|c|}{ Control group } \\
\hline & $\begin{array}{l}\text { MA (+) } \\
(n=45)\end{array}$ & $\begin{array}{l}\text { MA (-) } \\
(n=50)\end{array}$ & $p^{*}$ & $\begin{array}{l}\text { Overall } \\
(n=95)\end{array}$ & $(n=91)$ & $p^{* *}$ & $p^{* * *}$ \\
\hline Age (years) & $53.2 \pm 11.6$ & $53.8 \pm 9.1$ & 0.790 & $53.3 \pm 10.4$ & $53.8 \pm 8.8$ & 0.939 & 0.833 \\
\hline Gender $(\mathbf{F} / \mathbf{M})$ & $25 / 20$ & $31 / 19$ & 0.539 & $56 / 39$ & $48 / 43$ & 0.570 & 0.461 \\
\hline $\begin{array}{l}\text { DM duration } \\
\text { (years) }\end{array}$ & $9.5 \pm 5.4$ & $8.4 \pm 5.8$ & 0.411 & $8.9 \pm 5.6$ & N/A & N/A & N/A \\
\hline HbA1c (\%) & $7.2 \pm 1.4$ & $7.1 \pm 1.1$ & 0.600 & $7.1 \pm 1.2$ & N/A & N/A & N/A \\
\hline
\end{tabular}

All quantitative values were given as mean \pm standard deviation (SD)

$D M$ diabetes mellitus, $F / M$ female/male, $H b A l c$ glycosylated haemoglobin, $M A$ microalbuminuria, $N / A$ nonapplicable

*Comparison of age, gender, DM duration and HbA1c level between the MA (+) and MA (-) groups. Oneway ANOVA test with post hoc pairwise comparison (for quantitative variables) and chi-square test (for gender), $p<0.05$ indicates statistically significant difference

**Comparison of age and gender among the MA $(+)$, MA $(-)$ and control groups, one-way ANOVA test (for age) and chi-square test (for gender), $p<0.05$ indicates statistically significant difference

****Comparison of age and gender between the DM (total) and control groups, independent samples $t$-test (for quantitative variables) and chi-square test (for gender), $p<0.05$ indicates statistically significant difference terms of age and gender among the MA $(+)$, MA $(-)$ and control groups $(p=0.939$, one-way ANOVA test and $p=$ 0.570 , chi square test, respectively). Demographic and clinical details of the study groups were presented in Table 1.

There were no statistically significant differences in terms of SD-OCT measurements among the MA (+), MA $(-)$ and control groups ( $p>0.05$, one-way ANOVA test) (Table 2). On the other hand, when all diabetic patients $(n=95)$ were compared with the non-diabetic control subjects $(n=91)$, a significant decrease in inferior-temporal peripapillary RNFL thickness $(p=0.042$, independent samples $t$-test) was detected in the DM group, whereas remaining SD-OCT parameters did not differ between the DM and control groups (Table 2, $p>0.05$, independent samples $t$-test).

In patients with type $2 \mathrm{DM}$, mean duration of DM was $8.9 \pm 5.6$ years and mean $\mathrm{HbA} 1 \mathrm{c}$ level was $7.1 \pm 1.2 \%$. The MA (+) and MA (-) groups did not differ regarding DM duration ( $p=0.411$, independent samples $t$-test) and HbA1c levels ( $p=0.600$, independent samples $t$-test) (Table 1$)$.

When the diabetic patients were grouped according to the HbA1c level as $\leq 7 \%$ and $>7 \%$, superior RNFL was found to be significantly thinner in patients with HbAlc level $>7 \%$ than in the subjects with a HbA1c level $\leq 7 \%(p=0.049$, independent samples $t$-test), whereas other peripapillary and macular SD-OCT measurements did not differ between the two groups $(p>0.05$, independent samples $t$-test $)$ (Fig. 1).

The subgroup analysis based on DM duration demonstrated that superior, nasal and temporal perifoveal thickness values in the $3 \mathrm{~mm}$ ETDRS circle were significantly lower in patients with DM duration $>10$ years compared with those of the patients with DM duration $\leq 10$ years $(p=$ $0.015, p=0.006$ and $p=0.021$, respectively, independent samples $t$-test), while remaining SD-OCT parameters were similar between these two groups $(p>0.05$, independent samples $t$-test) (Fig. 2). On the other hand, mean age, MV and $\mathrm{CV}$ were statistically similar between the subgroups based on HbAlc level ( $\leq 7 \%$ vs. $>7 \%$ ) and DM duration ( $\leq 10$ years vs. $>10$ years) $(p>0.05)$.

The diabetic patients were also grouped according to existing systemic comorbidities as group 1 (MA-, HT and/ or $\mathrm{HL}-, n=25)$, group 2 (MA - , HT and/or $\mathrm{HL}+, n=25$ ), group $3(\mathrm{MA}+$, $\mathrm{HT}$ and/or $\mathrm{HL}-, n=15)$ and group $4(\mathrm{MA}+, \mathrm{HT}$ and/or $\mathrm{HL}+, n=30)$ to compare SD-OCT measurements. However, no significant differences were detected regarding SD-OCT parameters among these four groups ( $p>0.05$, Kruskal-Wallis test). On the other hand, subjects in group 3 were significantly younger than that of the group $4(45.4 \pm 8.9$ vs. $57.2 \pm 10.9$ years, respectively, $p=0.001$, Kruskal-Wallis test with Bonferroni correction).

Correlation analysis revealed statistically significant relations among age, DM duration, HbA1c level and macular measurements (centre and in the $3 \mathrm{~mm}$ of the ETDRS circle) $(p<0.05$, Pearson correlation coefficients) (Table 3). However, peripapillary SD-OCT parameters were not significantly correlated with any other variable $(p>$ 0.05 , Pearson correlation coefficients).

\section{Discussion}

Growing evidence points out neurodegenerative component of diabetic retinal disease [8-10, 12, 13]. Furthermore, several publications suggested that retinal 
Table 2 Comparison of macular and peripapillary spectral domain optical coherence tomography (SD-OCT) parameters among the microalbuminuria $(+)$, microalbuminuria (-) and control groups. Comparisons between DM (overall) and control groups were also presented

\begin{tabular}{|c|c|c|c|c|c|c|}
\hline \multirow[b]{2}{*}{ SD-OCT parameters } & \multicolumn{3}{|l|}{ DM group } & \multicolumn{3}{|c|}{ Control group } \\
\hline & $\mathrm{MA}(+)(n=45)$ & MA $(-)(n=50)$ & Overall $(n=95)$ & $(n=91)$ & $p^{*}$ & $p^{* *}$ \\
\hline \multicolumn{7}{|l|}{ Macular } \\
\hline $\mathrm{MV}\left(\mathrm{mm}^{3}\right)$ & $8.60 \pm 0.41$ & $8.52 \pm 0.50$ & $8.56 \pm 0.46$ & $8.61 \pm 0.40$ & 0.549 & 0.461 \\
\hline $\mathrm{CV}\left(\mathrm{mm}^{3}\right)$ & $0.20 \pm 0.01$ & $0.21 \pm 0.02$ & $0.20 \pm 0.01$ & $0.21 \pm 0.02$ & 0.314 & 0.666 \\
\hline $\mathrm{CT}(\mu \mathrm{m})$ & $264.8 \pm 22.8$ & $267.8 \pm 24.9$ & $266.4 \pm 23.9$ & $267.2 \pm 27.5$ & 0.830 & 0.839 \\
\hline IT-3mm $(\mu \mathrm{m})$ & $336.3 \pm 21.3$ & $329.9 \pm 37.4$ & $333.0 \pm 30.8$ & $338.0 \pm 16.3$ & 0.176 & 0.165 \\
\hline $\mathrm{ST}-3 \mathrm{~mm}(\mu \mathrm{m})$ & $343.2 \pm 16.6$ & $337.7 \pm 20.1$ & $340.3 \pm 18.8$ & $342.6 \pm 27.3$ & 0.412 & 0.510 \\
\hline NT-3mm $(\mu \mathrm{m})$ & $341.2 \pm 17.8$ & $337.2 \pm 24.6$ & $339.1 \pm 21.6$ & $340.5 \pm 17.1$ & 0.537 & 0.621 \\
\hline TT-3mm $(\mu \mathrm{m})$ & $325.8 \pm 34.1$ & $325.3 \pm 20.1$ & $325.6 \pm 27.5$ & $330.9 \pm 21.8$ & 0.341 & 0.143 \\
\hline \multicolumn{7}{|l|}{ Peripapillary } \\
\hline IRNFL $(\mu \mathrm{m})$ & $129.2 \pm 17.7$ & $131.0 \pm 19.3$ & $130.2 \pm 36.3$ & $130.2 \pm 21.0$ & 0.957 & 0.988 \\
\hline SRNFL $(\mu \mathrm{m})$ & $122.9 \pm 17.4$ & $125.2 \pm 16.4$ & $124.1 \pm 16.8$ & $127.0 \pm 18.7$ & 0.461 & 0.279 \\
\hline NRNFL $(\mu \mathrm{m})$ & $79.4 \pm 17.7$ & $78.3 \pm 12.8$ & $78.8 \pm 15.2$ & $77.7 \pm 12.6$ & 0.797 & 0.575 \\
\hline TRNFL $(\mu \mathrm{m})$ & $72.9 \pm 17.0$ & $71.0 \pm 13.0$ & $71.9 \pm 15.0$ & $73.2 \pm 11.8$ & 0.639 & 0.503 \\
\hline GRNFL $(\mu \mathrm{m})$ & $99.3 \pm 11.1$ & $100.8 \pm 11.3$ & $100.1 \pm 11.1$ & $102.3 \pm 10.8$ & 0.339 & 0.189 \\
\hline INRNFL $(\mu \mathrm{m})$ & $111.8 \pm 27.4$ & $118.9 \pm 22.4$ & $115.6 \pm 25.0$ & $114.9 \pm 23.8$ & 0.366 & 0.859 \\
\hline ITRNFL $(\mu \mathrm{m})$ & $134.7 \pm 30.8$ & $143.1 \pm 28.2$ & $139.2 \pm 29.6$ & $152.2 \pm 53.8$ & 0.083 & 0.042 \\
\hline SNRNFL $(\mu \mathrm{m})$ & $107.2 \pm 19.1$ & $112.4 \pm 21.1$ & $110.0 \pm 20.3$ & $111.1 \pm 21.5$ & 0.459 & 0.711 \\
\hline STRNFL $(\mu \mathrm{m})$ & $136.3 \pm 18.6$ & $137.9 \pm 21.1$ & $137.1 \pm 19.9$ & $142.9 \pm 20.5$ & 0.152 & 0.057 \\
\hline
\end{tabular}

All quantitative values were given as mean \pm standard deviation (SD)

$D M$ diabetes mellitus, $M A$ microalbuminuria, $\mu m$ microns, $3 \mathrm{~mm} 3 \mathrm{~mm}$ ETDRS circle, $C T$ centre thickness, $C V$ centre volume, GRNFL global retinal nerve fibre layer thickness (RNFL), INRNFL inferior-nasal peripapillary RNFL, IRNFL inferior peripapillary RNFL, IT inferior perifoveal thickness, ITRNFL inferiortemporal peripapillary RNFL, $M V$ macular volume, $N R N F L$ nasal peripapillary RNFL, $N T$ nasal perifoveal thickness, SNRNFL superior-nasal peripapillary RNFL, SRNFL superior peripapillary RNFL, $S T$ superior perifoveal thickness, STRNFL superior-temporal peripapillary RNFL, TT temporal perifoveal thickness, TRNFL temporal peripapillary RNFL

*Comparison of the MA (+), MA (-) and control groups, one-way ANOVA test with Bonferroni correction, $p<0.05$ indicates statistically significant difference

**Comparison of DM (overall) and control groups, independent samples $t$-test, $p<0.05$ indicates statistically significant difference neurodegeneration could be present in preclinical DR phase and it might precede the vascular retinal disease [8, 9, 14].

On the other hand, MA, which reflects generalized endothelial dysfunction, was found to be associated with presence or worsening of vascular DR in type 1 DM. However, in patients with type $2 \mathrm{DM}$, clinical evidence is not robust enough to suggest a direct link between MA and DR. Studies recommend a multifactorial approach including age, DM duration, HbA1c level, MA and HL and/or HT coexistence to predict DR [4-6, 15-17].

The main objective of the current study was to demonstrate effects of MA, HT and/or HL, DM duration and HbA1c level on neuroretinal alterations in macular and peripapillary regions in patients with preclinical DR. On the other hand, there is only one study reporting effect of MA on retinal layers in preclinical DR [11]. In our study, MA $(+)$ diabetic patients had similar SD-OCT measurements when compared with MA (-) diabetic subjects and nondiabetic healthy controls. Ucgul Atilgan et al. [11] presented the only similar study to ours and they found significant differences in superior, inferior and nasal parafoveal thickness values among patients without DR in MA stage (group 1), patients with mild DR in MA stage (group 2) and patients without DR and MA (group 3). However, they also stated that significance was originated from differences between group 2 (had lower values) and group 3. The differences in macular OCT measurements between the group 1 and group 3 were also non-significant as in our study [11].

In the current literature, studies presented different results regarding alterations in retinal layers in diabetic patients without DR. For instance, Demir et al. [18] and Oshitari et al. [19] agreed that diabetic patients without DR had significantly thinner macula than that of control eyes. Another study by Vujosevic et al. [20] showed that internal limiting membrane (+) RNFL thickness was significantly decreased in the non-DR group compared with those of the control group. On the contrary, Van Dijk et al. [21] and Zeng et al. [22] reported no significant difference in macular 


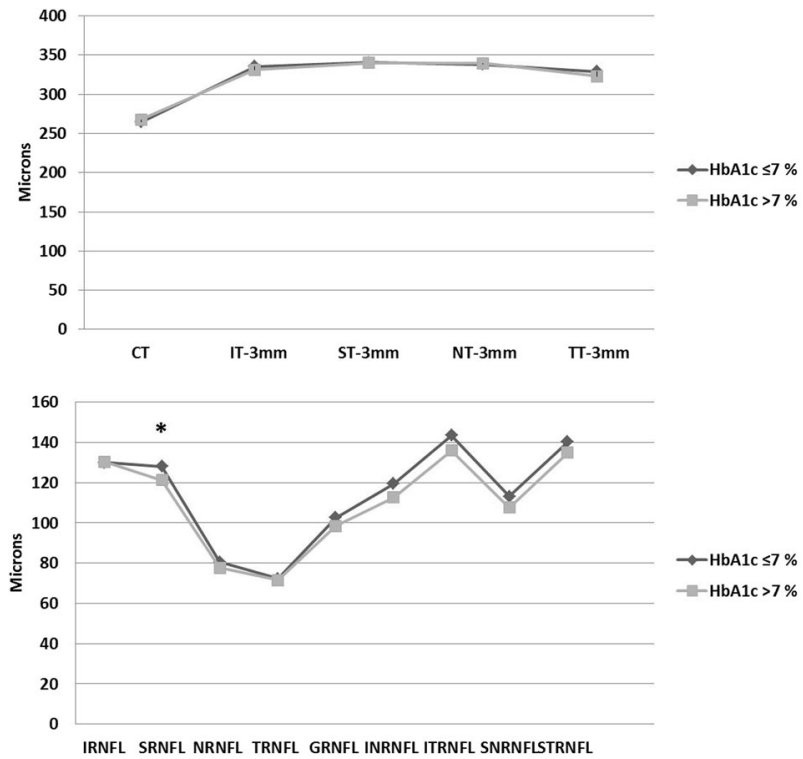

Fig. 1 The graph (top) shows similar central foveal thickness (CT), inferior (IT), superior (ST), nasal (NT) and temporal (TT) macular thickness (microns) values (within the $3 \mathrm{~mm}$ macular ETDRS circle) between the diabetic patients with $\mathrm{HbA} 1 \mathrm{c}$ level $\leq 7 \%$ vs. HbA1c $>7 \%$ $(p=0.482, p=0.501, p=0.905, p=0.746$ and $p=0.307$, respectively, independent samples $t$-test). The peripapillary superior retinal nerve fibre layer (SRNFL) was significantly thinner $(p=0.049)$ in patients with $\mathrm{HbA} 1 \mathrm{c}>7 \%$ when compared with the patients with HbA1c level $\leq 7 \%$. However, inferior (IRNFL), nasal (NRNFL), temporal (TRNFL), global (GRNFL), inferior-nasal (INRNFL), inferior-temporal (ITRNFL), superior-nasal (SNRNFL) and superiortemporal (STRNFL) peripapillary RNFL thicknesses did not differ between the two groups (bottom graph) ( $p=0.982, p=0.371, p=$ $0.847, p=0.063, p=0.193, p=0.233, p=0.191$ and $p=0.191$, respectively). An asterisk indicates statistically significant difference between the two groups $(p<0.05)$, independent samples $t$-test

ganglion cell layer thickness between diabetic patients without DR and controls. Consequently, results of our study agree with the studies reporting similar macular OCT measurements between the non-DR diabetic patients and controls.

In our study, inferior-temporal peripapillary RNFL was significantly thinner in the non-DR group than in the control group (for about $-13 \mu \mathrm{m}$ ), while remaining RNFL thickness values did not differ between the two groups. Vujosevic et al. [23] reported a similar decrease in inferior peripapillary RNFL thickness in patients without DR compared with that of the non-diabetic controls. A metaanalysis by Chen et al. [24] demonstrated that peripapillary average RNFL thickness was significantly reduced (for about $-2.88 \mu \mathrm{m}$ ) in the non-DR group when compared with the non-diabetic controls based on SD-OCT measurements. They also stated that reduction in RNFL thickness was more significant in the superior quadrant $(-3.79 \mu \mathrm{m})$, inferior quadrant $(-2.99 \mu \mathrm{m})$ and nasal quadrant $(-2.88 \mu \mathrm{m})$ in order. Similarly, Peng et al. [25] presented that mean and
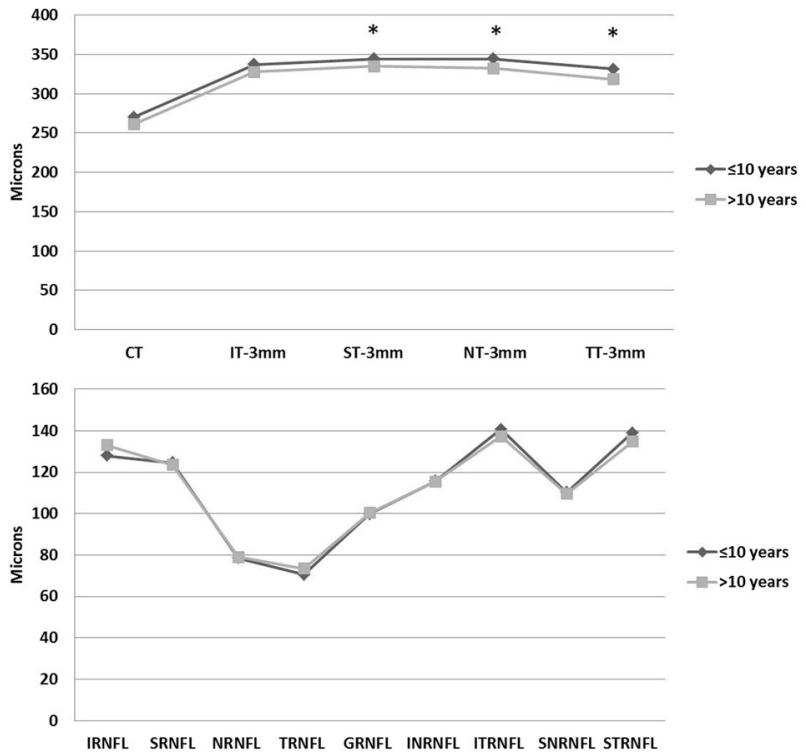

Fig. 2 In patients with DM duration over 10 years, superior (ST, $p=$ $0.015)$, nasal (NT, $p=0.006)$ and temporal (TT, $p=0.021)$ macular thickness (microns) values (within the $3 \mathrm{~mm}$ ETDRS circle) were significantly lower when compared with that of the patients with DM duration $\leq 10$ years. However, central foveal thickness (CT) and inferior perifoveal thickness (IT) were similar between the two groups (top graph) ( $p=0.071$ and $p=0.157$, respectively). There were no statistically significant differences regarding peripapillary inferior (IRNFL), superior (SRNFL), nasal (NRNFL), temporal (TRNFL), global (GRNFL), inferior-nasal (INRNFL), inferior-temporal (ITRNFL), superior-nasal (SNRNFL) and superior-temporal (STRNFL) RNFL thicknesses between patients with DM duration over 10 years and patients with DM duration $\leq 10$ years (bottom graph) $(p=0.496, p=0.742, p=0.878, p=0.366, p=0.853, p=0.975$, $p=0.586, p=0,879$ and $p=0.320$, respectively, independent samples t-test). An asterisk indicates statistically significant difference between the two groups $(p<0.05)$, independent samples $t$-test

superior quadrant peripapillary RNFL thicknesses were decreased in diabetic patients without DR than in the healthy controls. Shi et al. [26] also reported significant differences regarding average, superior and inferior RNFL thicknesses between non-DR and moderate nonproliferative DR groups. However, the mentioned study had no non-diabetic control group and failed to demonstrate whether there was a significant difference between patients without DR and non-diabetic controls in contrast to our study. Moreover, our study partially disagrees with the above-mentioned studies, because we found comparable average, superior and nasal peripapillary RNFL thicknesses between non-DR diabetic subjects and controls in our study; on the other hand, inferior-temporal sector RNFL was affected.

In the current study, we also investigated effects of DM duration, HbA1c level, MA and coincidence of HT and/or HL on SD-OCT parameters, since a multifactorial assessment is recommended in patients with type $2 \mathrm{DM}$ to precede diabetic retinal disease. However, presence of 
Table 3 Correlations among age, DM duration, HbA1c level and macular optical coherence tomography (SD-OCT) parameters in the DM group

\begin{tabular}{|c|c|c|c|c|c|c|c|c|c|c|c|}
\hline & & \multirow[t]{2}{*}{ Age } & \multirow[t]{2}{*}{ DM duration } & \multirow[t]{2}{*}{ HbA1c } & \multicolumn{7}{|c|}{ Macular SD-OCT parameters } \\
\hline & & & & & MV & $\mathrm{CT}$ & $\mathrm{CV}$ & NT $(3 \mathrm{~mm})$ & IT (3 mm) & TT (3 mm) & $\mathrm{ST}(3 \mathrm{~mm})$ \\
\hline \multirow[t]{2}{*}{ Age } & $r$ & 1 & $0.365^{*}$ & 0.155 & $-0.284^{*}$ & -0.086 & -0.089 & -0.191 & $-0.302^{*}$ & $-0.246^{*}$ & $-0.322^{*}$ \\
\hline & $p$ & & 0.001 & 0.171 & 0.005 & 0.408 & 0.394 & 0.063 & 0.003 & 0.016 & 0.001 \\
\hline \multirow[t]{2}{*}{ DM duration } & $r$ & $0.365^{* *}$ & 1 & $0.382^{* * *}$ & $-0.325^{*}$ & $-0.315^{*}$ & $-0.321 *$ & $-0.420^{*}$ & $-0.315^{*}$ & $-0.375^{*}$ & $-0.441^{*}$ \\
\hline & $p$ & 0.001 & & 0.001 & 0.004 & 0.006 & 0.005 & 0.000 & 0.006 & 0.001 & $<0.0001$ \\
\hline \multirow[t]{2}{*}{ HbA1c } & $r$ & 0.155 & $0.382^{*}$ & 1 & -0.054 & -0.019 & -0.030 & -0.023 & -0.015 & -0.122 & -0.052 \\
\hline & $p$ & 0.171 & 0.001 & & 0.635 & 0.866 & 0.789 & 0.837 & 0.897 & 0.282 & 0.647 \\
\hline
\end{tabular}

$D M$ diabetes mellitus, $H b A l c$ glycosylated haemoglobin (\%), $3 \mathrm{~mm} 3 \mathrm{~mm}$ ETDRS circle, $C T$ centre thickness (microns), $C V$ centre volume $\left(\mathrm{mm}^{3}\right), I T$ inferior perifoveal thickness (microns), $M V$ macular volume $\left(\mathrm{mm}^{3}\right), N T$ nasal perifoveal thickness (microns), $S T$ superior perifoveal thickness (microns), TT temporal perifoveal thickness (microns)

${ }^{*} p<0.05$ and bold values indicate statistically significant difference $(r=$ Pearson correlation coefficient, age $=$ years, DM duration $=$ years $)$

HT and/or HL with or without MA did not affect macular and peripapillary SD-OCT measurements in the DM group. On the other hand, superior peripapillary RNFL was significantly thinner in patients with a higher $\mathrm{HbA} 1 \mathrm{c}$ level (>7\% vs. $\leq 7 \%)$, while remaining macular and peripapillary SD-OCT parameters did not differ between the two groups. On the contrary, nasal, temporal and superior macular thickness values in the $3 \mathrm{~mm}$ ETDRS circle were significantly lower in patients with a longer DM duration ( $>10$ years vs. $\leq 10$ years). In a similar study, Tavares Ferreira et al. [27] performed a subgroup analysis as we did in diabetic patients without DR according to DM duration as group $1(<5$ years), group 2 (5-10 years) and group 3 (>10 years). They found that subjects in group 1 and group 3 had thinner photoreceptor layer (PRL) thickness, when compared with that of the non-diabetic controls. Moreover, group 1 had lower PRL thickness than those of the group 2.

Correlation analyses in our study showed that DM duration had a weak negative relation with macular SDOCT measurements and a weak positive association with HbAlc level. On the other hand, MV, inferior, temporal and superior macular thicknesses were negatively correlated with age. Interestingly, HbAlc level had no significant correlation with any SD-OCT parameter in our study. Similarly, Demir et al. [18] detected no statistically significant relationship among central macular thickness, $\mathrm{HbA1c}$ and fasting plasma glucose levels in diabetic patients as in our study. On the contrary, Ucgul Atilgan et al. [11] found significant negative correlations between the parafoveal thickness values and serum urea, HbA1c, albumin and MA levels.

In terms of RNFL measurements, Peng et al. [25] reported a significant weak negative correlation between fasting blood glucose and RNFL thickness values in diabetic patients without DR. Shi et al. [26] suggested that DM duration, body mass index, triglyceride, high density lipoprotein cholesterol, $\mathrm{HbAlc}$ levels and albumin to creatinine ratio were negatively correlated with peripapillary RNFL thickness. However, in our study both DM duration and $\mathrm{HbA1c}$ levels were not correlated with peripapillary RNFL measurements. As discussed above, alterations in macular and peripapillary retinal layers and their correlation with DM duration and metabolic control remain controversial in diabetic patients without DR.

In our study, diabetic patients without DR had thinner inferior-temporal peripapillary RNFL and similar macular measurements when compared with those of the control group. Decrease in the peripapillary RNFL thickness could be an indicator of early retinal neurodegeneration and might be explained by two possible mechanisms based on the current knowledge: direct toxicity of hyperglycaemiainduced molecules, and microvascular insufficiency. In vitro studies support the first hypothesis that hyperglycaemia-induced toxic molecules cause Müller cell dysfunction, retinal ganglion cell damage and RNFL thinning [4-6]. It was also suggested that retinal ganglion cells and amacrine cells might be the first to succumb to hyperglycaemia induced apoptosis. However, clinical studies failed to demonstrate correspondence between ganglion cell damage and RNFL thinning. From microvascular perspective, it was suggested that inner retinal layers have high metabolic activity and relatively lower circulation. A study by Vusojevic et al. [23] demonstrated early deterioration in peripapillary superficial capillary plexus in diabetic patients without DR and they also documented correlations between these alterations and RNFL thinning. Interestingly, changes in superficial vessel density were manifested in the peripapillary region earlier than in the macular region [23]. They also suggested that this susceptibility might be due to specific anatomic characteristics of the superficial capillary plexus. Therefore, deterioration in retinal microcirculation in DM seems to affect inner retinal layers and peripapillary region initially. On the other hand, in the current study, we investigated effect of MA as a vascular endothelial dysfunction biomarker, whereas retinal neurodegenerative 
changes were found regardless from MA positivity (also coexistent HT or HL). Therefore, in early retinal neurodegenerative process in preclinical DR, hyperglycaemia induced toxicity could be more dominant. Furthermore, decreased superior peripapillary RNFL thickness (about $-7 \mu \mathrm{m}$ ) in patients with poor blood glucose control (HbA1c value over 7\%) might support this idea. Interestingly, longer DM duration (over 10 years) seems to lead superior, nasal, and temporal perifoveal thinning, while there was no statistically significant difference regarding age between the subgroups based on DM duration. On the other hand, correlation analysis revealed a weak negative association between age and macular SD-OCT measurements.

In conclusion, early retinal neurodegeneration appears to manifest as peripapillary RNFL thinning on SD-OCT prior to the retinal and generalized vascular deterioration, and it seems to be associated with poor blood glucose control. Therefore, strict HbA1c control $(\leq 7 \%)$ might prevent this neurodegenerative process in clinical practice. However, it is still unclear and needs to be elucidated why a particular region is affected in diabetic patients without DR regarding DM duration and HbA1c levels.

\section{Summary}

\section{What was known before}

- Retinal neurodegeneration is suggested to be involved in diabetic retinal disease. In the present literature, there is only one study reporting effect of MA on retinal layers in diabetic patients without retinopathy (preclinical DR).

\section{What this study adds}

- Peripapillary RNFL thinning in optical coherence tomography might be an early sign of neuroretinal degeneration in preclinical DR Neuroretinal degeneration seems to manifest prior to retinal (DR) and generalized (MA) vascular endothelial deterioration. Longer duration of DM appears to effect perifoveal thicknesses independent from age.

Acknowledgements The authors thank Hande Senol (from Department of Biostatistics) for statistical assistance. This study was supported by Pamukkale University Scientific Research Projects Coordination Unit under grantnumber 2018HZDP042 and 2019KRM004-022.

\section{Compliance with ethical standards}

Conflict of interest The authors declare that they have no conflict of interest.
Publisher's note Springer Nature remains neutral with regard to jurisdictional claims in published maps and institutional affiliations.

\section{References}

1. Forbes JM, Cooper ME. Mechanisms of diabetic complications. Physiol Rev. 2013;93:137-88. https://doi.org/10.1152/physrev. 00045.2011

2. Choby B. Diabetes update: prevention and management of diabetes complications. FP Ess. 2017;456:36-40.

3. Adler AI, Stevens RJ, Manley SE, Bilous RW, Cull CA, Holman RR. Development and progression of nephropathy in type 2 diabetes: the United Kingdom Prospective Diabetes Study (UKPDS64). Kidney Int. 2003;63:225-32.

4. Ahmad T, Ulhaq I, Mawani M, Islam N. Microalbuminuria in type-2 diabetes mellitus; the tip of iceberg of diabetic complications. Pak J Med Sci. 2017;33:519-23.

5. Parving HH, Chaturvedi N, Viberti G, Mogensen CE. Does microalbuminuria predict diabetic nephropathy. Diabetes Care. 2002;25:406-7.

6. Satchell SC, Tooke JE. What is the mechanism of microalbuminuria in diabetes: a role for the glomerular endothelium? Diabetologia. 2008;51:714-25. https://doi.org/10.1007/s00125008-0961-8.

7. Martens RJ, Henry RM, Houben AJ, van der Kallen CJ, Kroon AA, Schalkwijk CG, et al. Capillary Rarefaction associates with albuminuria: The Maastricht Study. J Am Soc Nephrol. 2016;27:3748-57.

8. Kim K, Kim ES, Yu SY. Longitudinal relationship between retinal diabetic neurodegeneration and progression of diabetic retinopathy in patients with type 2 diabetes. Am J Ophthalmol. 2018;196:165-72.

9. Santos AR, Ribeiro L, Bandello F, Lattanzio R, Egan C, Frydkjaer-Olsen U, et al. Functional and structural findings of neurodegeneration in early stages of diabetic retinopathy: crosssectional analyses of baseline data of the EUROCONDOR project. Diabetes. 2017;66:2503-10.

10. Gundogan FC, Akay F, Uzun S, Yolcu U, Çağıltay E, Toyran S. Early neurodegeneration of the inner retinal layers in type 1 diabetes mellitus. Ophthalmologica. 2016;235:125-32. https://doi. org/10.1159/000442826.

11. Ucgul Atilgan C, Atilgan KG, Kosekahya P, Caglayan M, Sendul SY, Yilmazbas P. Effect of microalbuminuria on macular thickness in patients with type-2 diabetes mellitus. Eur J Ophthalmol. 2018. https://doi.org/10.1177/1120672118811256.

12. Vujosevic S, Muraca A, Alkabes M, Villani E, Cavarzeran F, Rossetti L, et al. Early microvascular and neural changes in patients with type 1 and type 2 diabetes mellitus without clinical signs of diabetic retinopathy. Retina. 2019;39:435-45. https://doi. org/10.1097/IAE.0000000000001990

13. Hafner J, Karst S, Sacu S, Scholda C, Pablik E, Schmidt-Erfurth $\mathrm{U}$. Correlation between corneal and retinal neurodegenerative changes and their association with microvascular perfusion in type II diabetes. Acta Ophthalmol. 2018. https://doi.org/10.1111/aos. 13938.

14. Sohn EH, van Dijk HW, Jiao C, Kok PH, Jeong W, Demirkaya N, et al. Retinal neurodegeneration may precede microvascular changes characteristic of diabetic retinopathy in diabetes mellitus. Proc Natl Acad Sci USA. 2016;113:E2655-64. https://doi.org/10. 1073/pnas.1522014113.

15. Bakris GL, Molitch M. Microalbuminuria as a risk predictor in diabetes: the continuing saga. Diabetes Care. 2014;37:867-75.

16. van Dijk HW, Kok PHB, Garvin M, Sonka M, Devries JH, Michels RP, et al. Selective loss of inner retinal layer thickness in 
type 1 diabetic patients with minimal diabetic retinopathy. Investig Ophthalmol Vis Sci. 2009;50:3404-9.

17. Chang $\mathrm{CH}$, Chuang LM. Microalbuminuria and diabetic retinopathy in type 2 diabetic patients: From risk association to risk prediction. J Diabetes Investig. 2013;4:42-4. https://doi.org/10. 1111/jdi.12023.

18. Demir M, Oba E, Sensoz H, Ozdal E. Retinal nerve fiber layer and ganglion cell complex thickness in patients with type 2 diabetes mellitus. Indian J Ophthalmol. 2014;62:719-20.

19. Oshitari T, Hanawa K, Adachi-Usami E. Changes of macular and RNFL thicknesses measured by Stratus OCT in patients with early stage diabetes. Eye. 2009;23:884-9.

20. Vujosevic S, Midena E. Retinal layers changes in human preclinical and early clinical diabetic retinopathy support early retinal neuronal and Müller cells alterations. J Diabetes Res. 2013;2013:905058.

21. van Dijk HW, Verbraak FD, Kok PH, Stehouwer M, Garvin MK, Sonka M, et al. Early neurodegeneration in the retina of type 2 diabetic patients. Investig Ophthalmol Vis Sci. 2012;53:2715-9.

22. Zeng Y, Cao D, Yu H, Yang D, Zhuang X, Hu Y, et al. Early retinal neurovascular impairment in patients with diabetes without clinically detectable retinopathy. Br J Ophthalmol. 2019. https:// doi.org/10.1136/bjophthalmol-2018-313582.

23. Vujosevic S, Muraca A, Gatti V, Masoero L, Brambilla M, Cannillo B, et al. Peripapillary microvascular and neural changes in diabetes mellitus: an OCT-Angiography study. Investig Ophthalmol Vis Sci. 2018;59:5074-81.

24. Chen X, Nie C, Gong Y, Zhang Y, Jin X, Wei S, et al. Peripapillary retinal nerve fiber layer changes in preclinical diabetic retinopathy: a meta-analysis. Plos ONE. 2015;10:e125919.

25. Peng PH, Lin HS, Lin S. Nerve fibre layer thinning in patients with preclinical retinopathy. Can J Ophthalmol. 2009;44:417-22. https://doi.org/10.3129/i09-112.

26. Shi R, Guo Z, Wang F, Li R, Zhao L, Lin R. Alterations in retinal nerve fiber layer thickness in early stages of diabetic retinopathy and potential risk factors. Curr Eye Res. 2018;43:244-53. https:// doi.org/10.1080/02713683.2017.1387669.

27. Tavares Ferreira J, Alves M, Dias-Santos A, Costa L, Santos BO, Cunha JP, et al. Retinal neurodegeneration in diabetic patients without diabetic retinopathy. Investig Ophthalmol Vis Sci. 2016;57:6455-60. https://doi.org/10.1167/iovs.16-20215. 